\title{
Supporting Information: Intramolecular Hydrogen Bonding in Substituted Aminoalcohols
}

Joseph R. Lane, ${ }^{* \dagger}$ Sidsel D Schrøder ${ }^{\ddagger}$ Graham C. Saunders, ${ }^{\dagger}$ and Henrik G. Kjaergaard $\ddagger$

$\dagger$ School of Science, Faculty of Science and Engineering, University of Waikato, Private Bag 3105, Hamilton, New Zealand

$\ddagger$ Department of Chemistry, University of Copenhagen, Universitetsparken 5, DK-2100

Copenhagen, Denmark.

E-mail: jlane@waikato.ac.nz

Phone: +64-7-837-9391 


\section{Additional computational details}

All DFT optimizations were completed using Gaussian $09,{ }^{1}$ with the Opt $=$ tight and Int $=$ UltraFine keywords to ensure that the optimized structures were accurately converged.

All CCSD(T)-F12a optimizations were completed using MOLPRO2012.1, ${ }^{2}$ with the following threshold criteria: gradient $=1 \times 10^{-6}$ a.u., stepsize $=1 \times 10^{-6}$ a.u., energy $=1 \times$ $10^{-8}$ a.u.

The necessary wavefunction files for the DFT electron densities were generated directly from Gaussian 09 using the output=wfn keyword. Single point CCSD(T)-F12a/cc-pVDZF12 electron densities were calculated using MOLPRO with all results converged to energy $=1 \times 10^{-10}$ a.u., orbital $=1 \times 10^{-9}$ a.u., coeff $=1 \times 10^{-9}$ a.u. All of the $\operatorname{CCSD}(\mathrm{T})-\mathrm{F} 12$ a calculations assume a frozen core (C:1s; O:1s; N:1s; F:1s) and were performed with the geminal Slater exponent $\beta$ set to $0.9 .^{3}$ The CCSD(T)-F12a/cc-pVDZ-F12 electron densities were exported from MOLPRO in MOLDEN format and subsequently converted to wavefunction format using the Molden2AIM program. ${ }^{4}$

\section{Additional results}




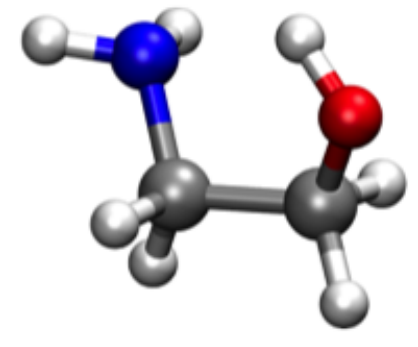

Conformer 1

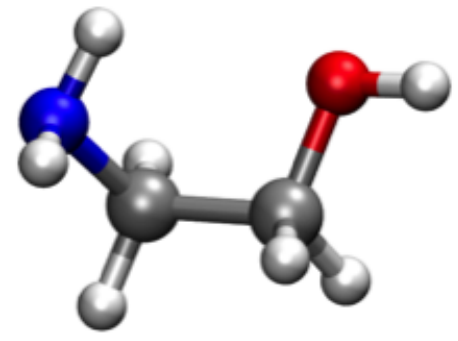

Conformer 3

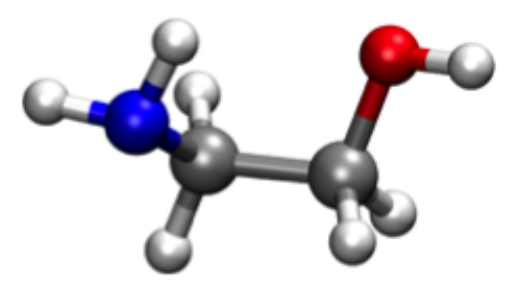

Conformer 2

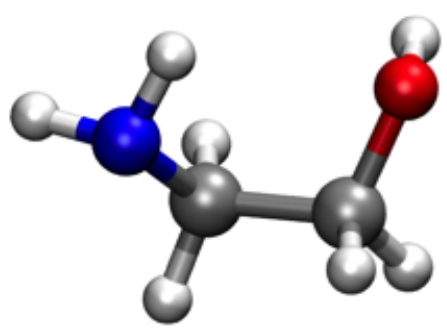

Conformer 4

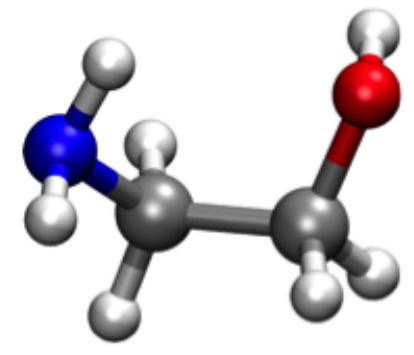

Conformer 5

Figure S1: The five lowest energy conformers of AE calculated with G4 theory. 


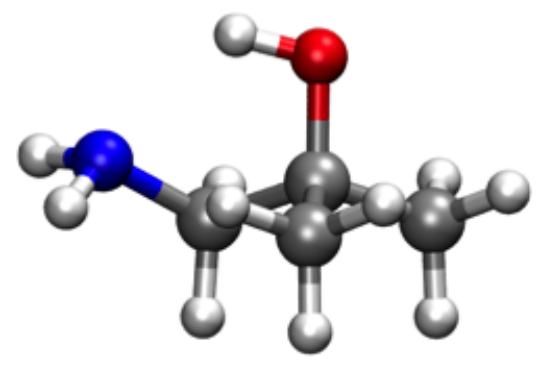

Conformer 1

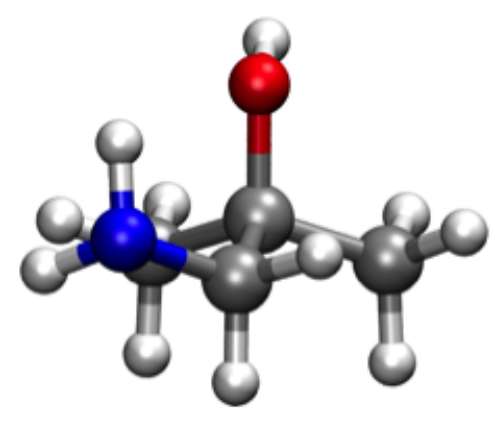

Conformer 3

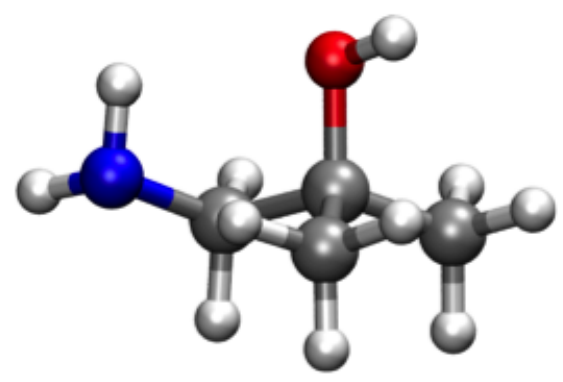

Conformer 2

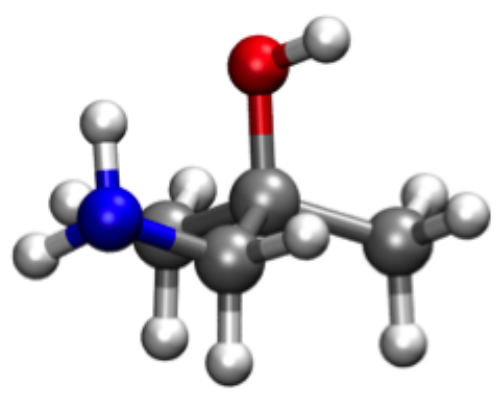

Conformer 4

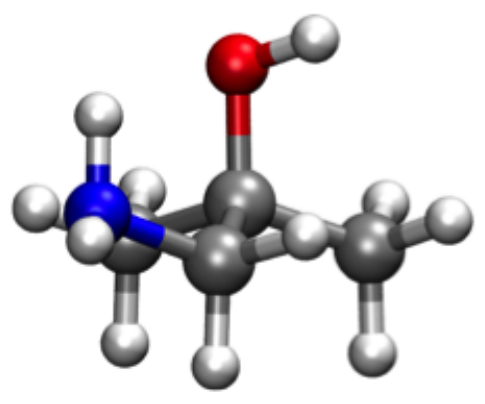

Conformer 5

Figure S2: The five lowest energy conformers of BMAE calculated with G4 theory. 


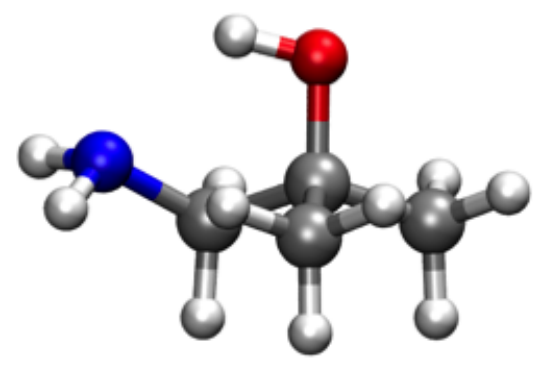

Conformer 1

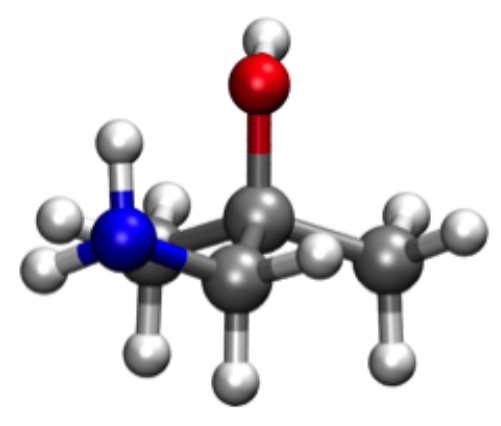

Conformer 3

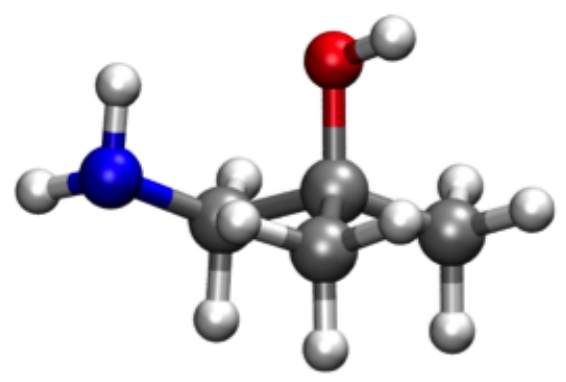

Conformer 2

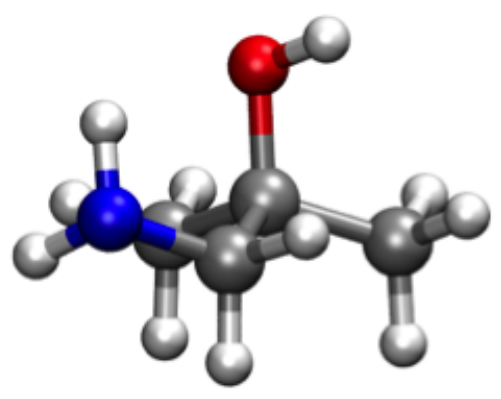

Conformer 4

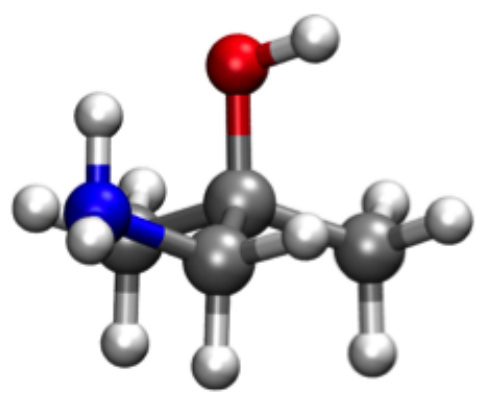

Conformer 5

Figure S3: The five lowest energy conformers of BFMAE calculated with G4 theory. 

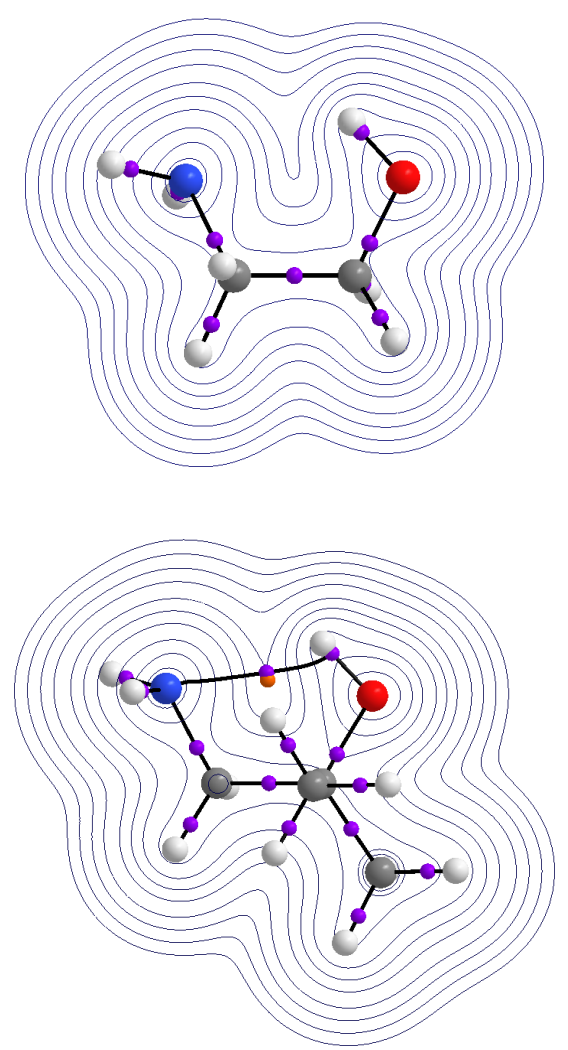

Figure S4: Electron density contour maps for AE (top), BMAE (bottom) obtained with the CCSD(T)-F12a/cc-VDZ-F12 method and optimised geometry. Small purple circles represent $(3,-1)$ BCPs, and small orange circles represent $(3,+1)$ RCPs. 

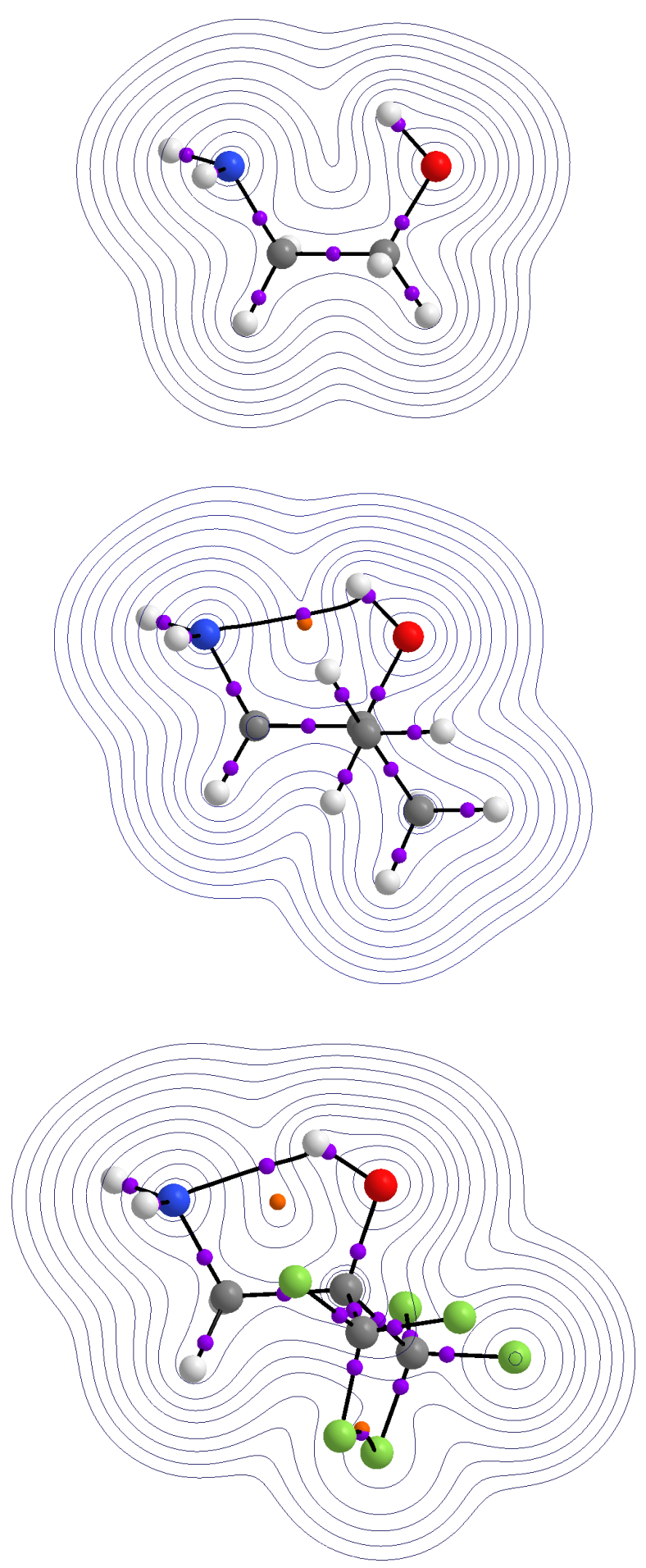

Figure S5: Electron density contour maps for AE (top), BMAE (middle) and BFMAE (bottom) obtained with the B3LYP/aug-cc-pVTZ method and optimised geometry. Small purple circles represent $(3,-1)$ BCPs, and small orange circles represent $(3,+1)$ RCPs. 

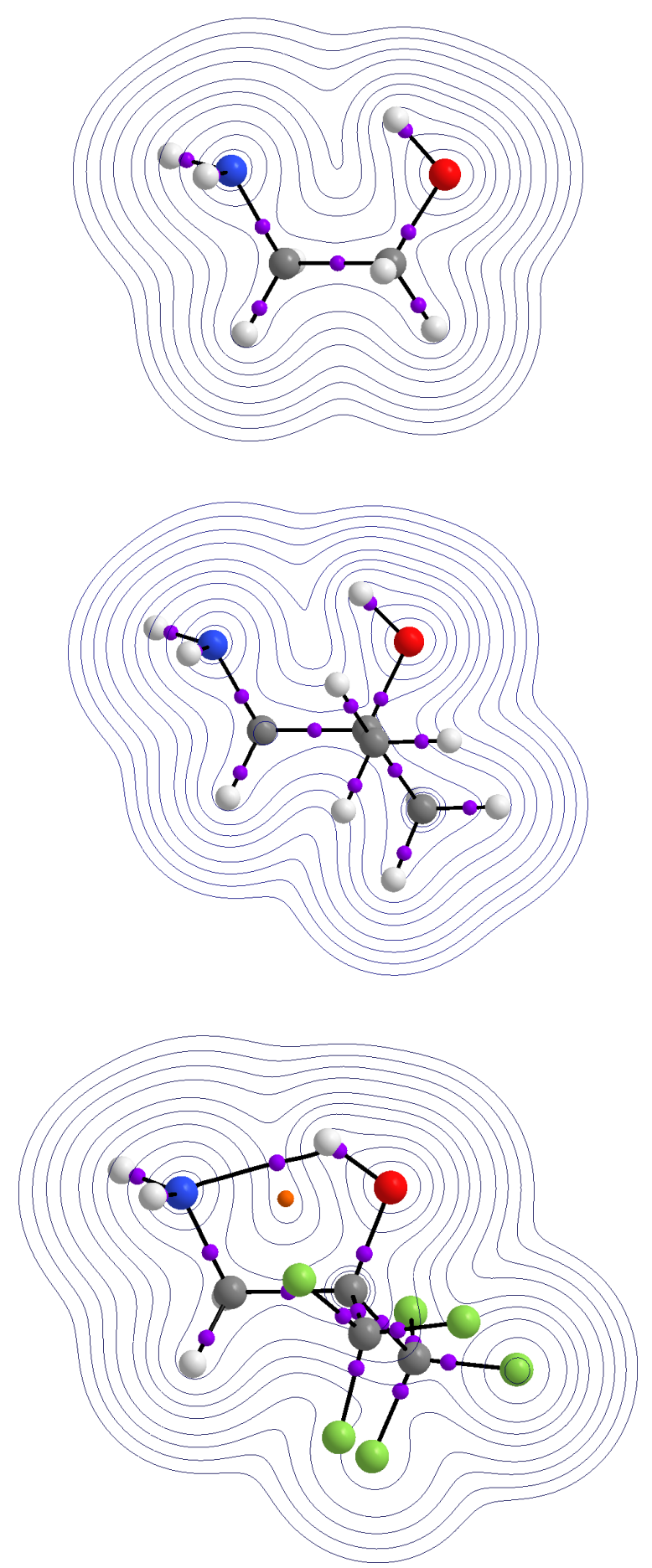

Figure S6: Electron density contour maps for AE (top), BMAE (middle) and BFMAE (bottom) obtained with the WB97XD/aug-cc-pVTZ method. Small purple circles represent $(3,-1) \mathrm{BCPs}$, and small orange circles represent $(3,+1)$ RCPs. 

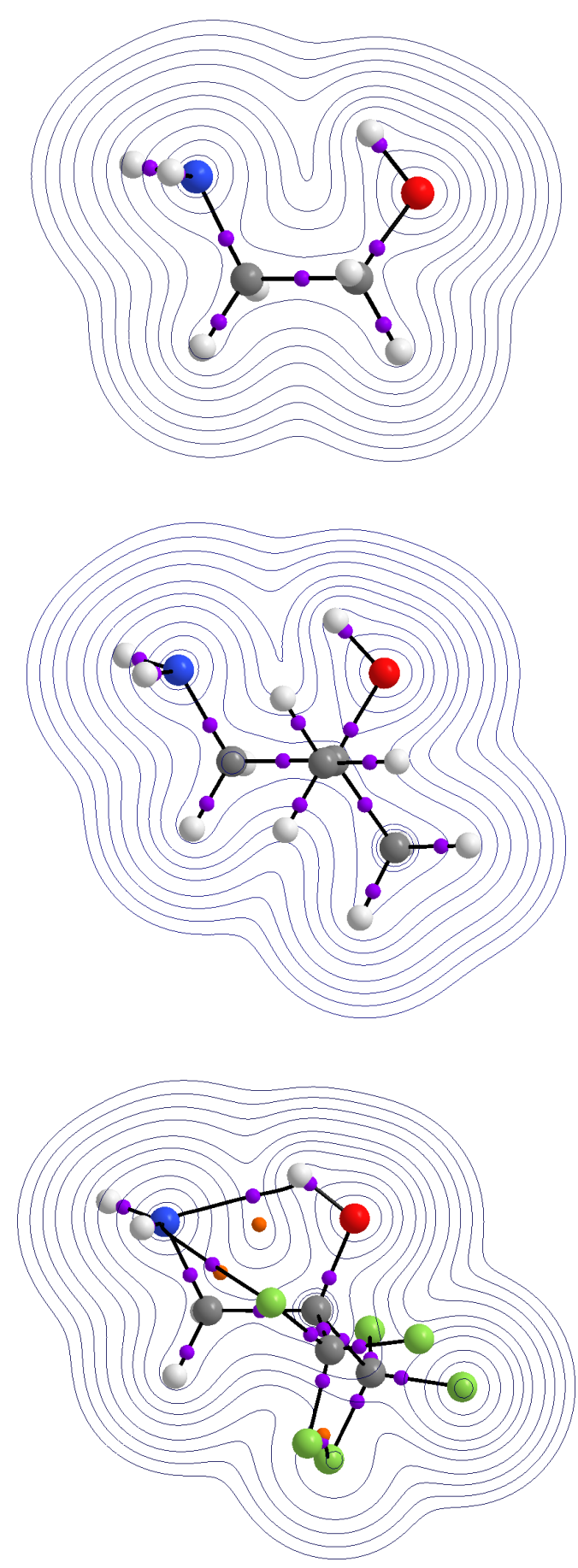

Figure S7: Electron density contour maps for AE (top), BMAE (middle) and BFMAE (bottom) obtained with the M06-2X/aug-cc-pVTZ method and optimised geometry. Small purple circles represent $(3,-1) \mathrm{BCPs}$, and small orange circles represent $(3,+1) \mathrm{RCPs}$. 

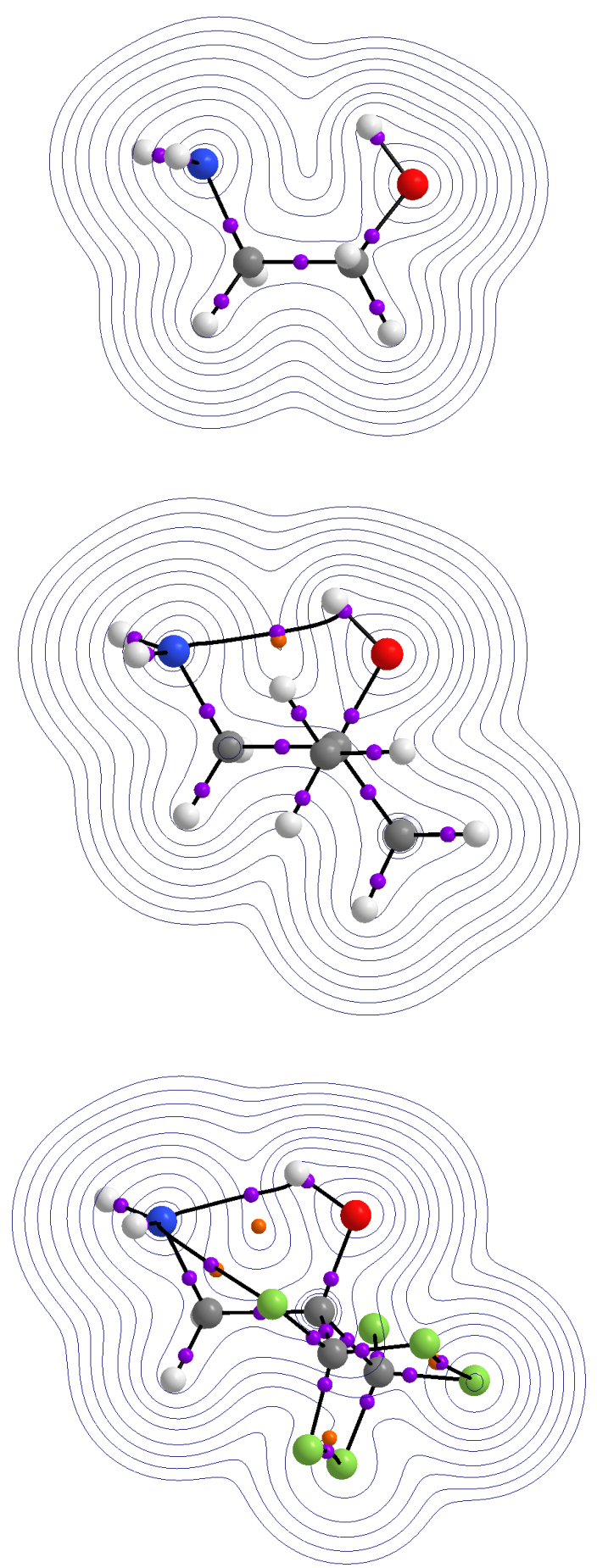

Figure S8: Electron density contour maps for AE (top), BMAE (middle) and BFMAE (bottom) obtained with the CCSD(T)-F12a/cc-pVDZ-F12 method using the B3LYP/augcc-pVTZ optimised geometry. Small purple circles represent $(3,-1)$ BCPs, and small orange circles represent $(3,+1)$ RCPs. 

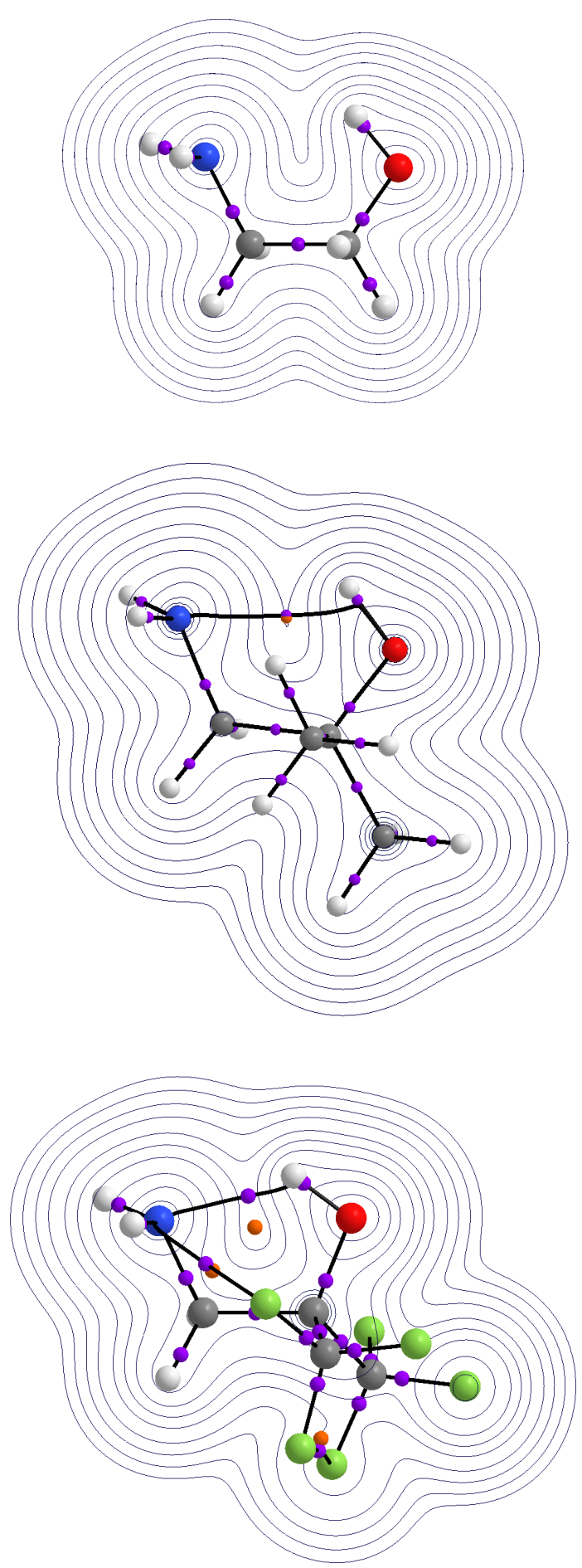

Figure S9: Electron density contour maps for AE (top), BMAE (middle) and BFMAE (bottom) obtained with the CCSD(T)-F12a/cc-pVDZ-F12 method using the wB97XD/augcc-pVTZ optimised geometry. Small purple circles represent $(3,-1)$ BCPs, and small orange circles represent $(3,+1)$ RCPs. 

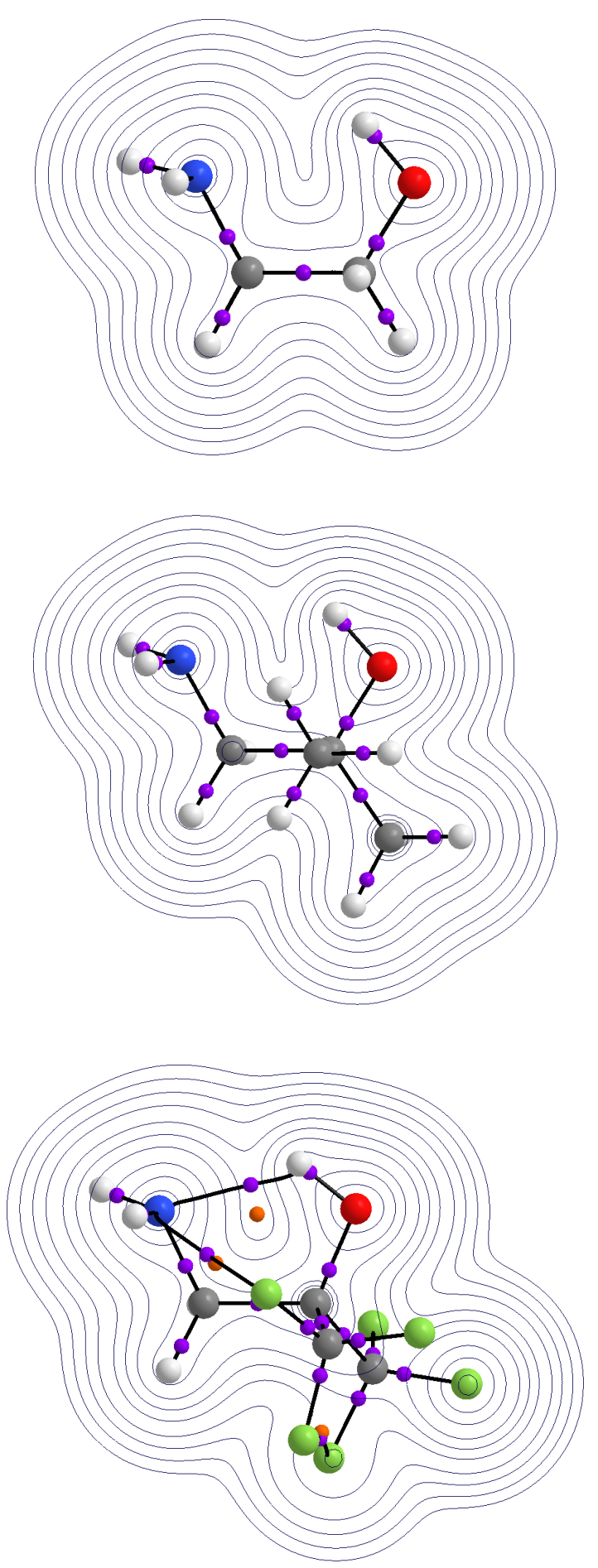

Figure S10: Electron density contour maps for AE (top), BMAE (middle) and BFMAE (bottom) obtained with the CCSD(T)-F12a/cc-pVDZ-F12 method using the M06-2X/augcc-pVTZ optimised geometry. Small purple circles represent $(3,-1)$ BCPs, and small orange circles represent $(3,+1)$ RCPs. 

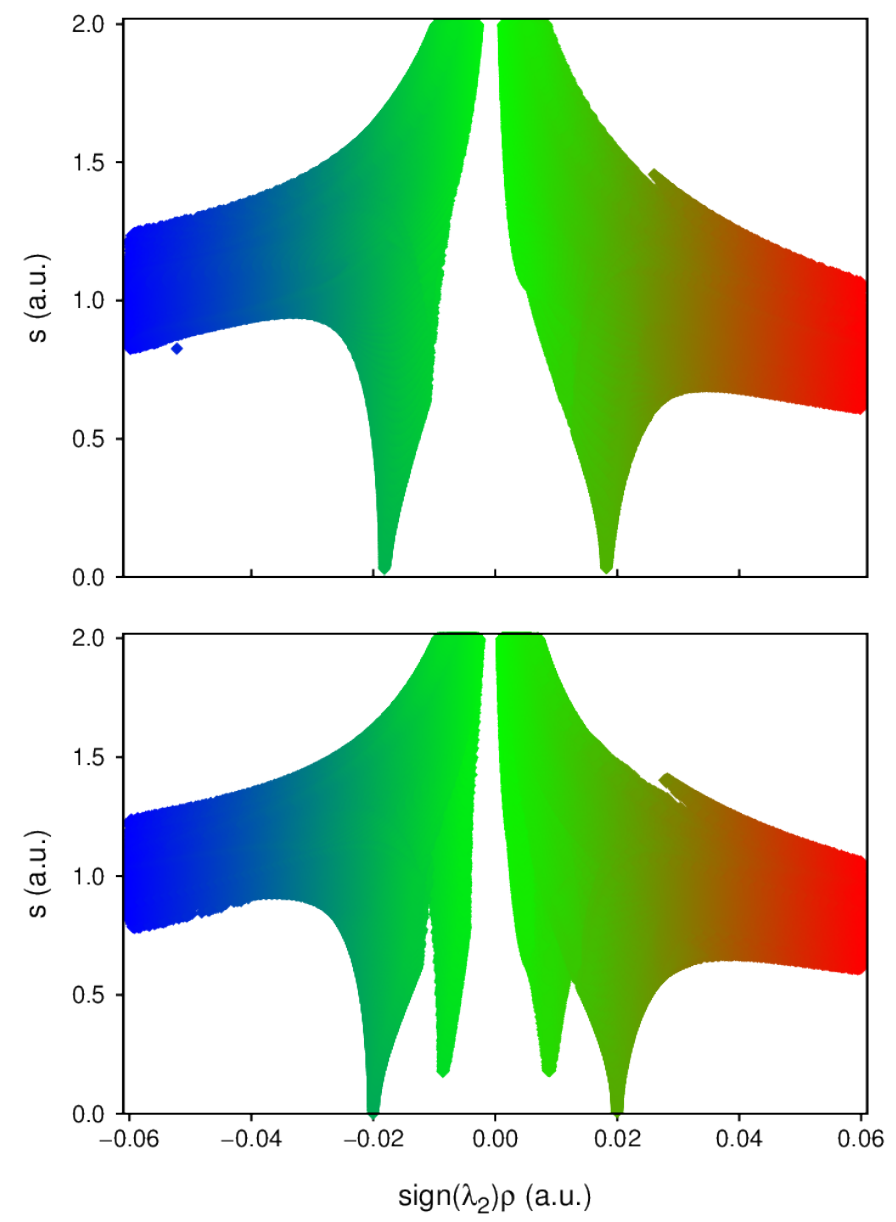

Figure S11: Plots of the reduced density gradient $s$ and $\operatorname{sign}\left(\lambda_{2}\right) \rho$ for AE (top) and BMAE (bottom) obtained with the CCSD(T)-F12a/cc-pVDZ-F12 method. 

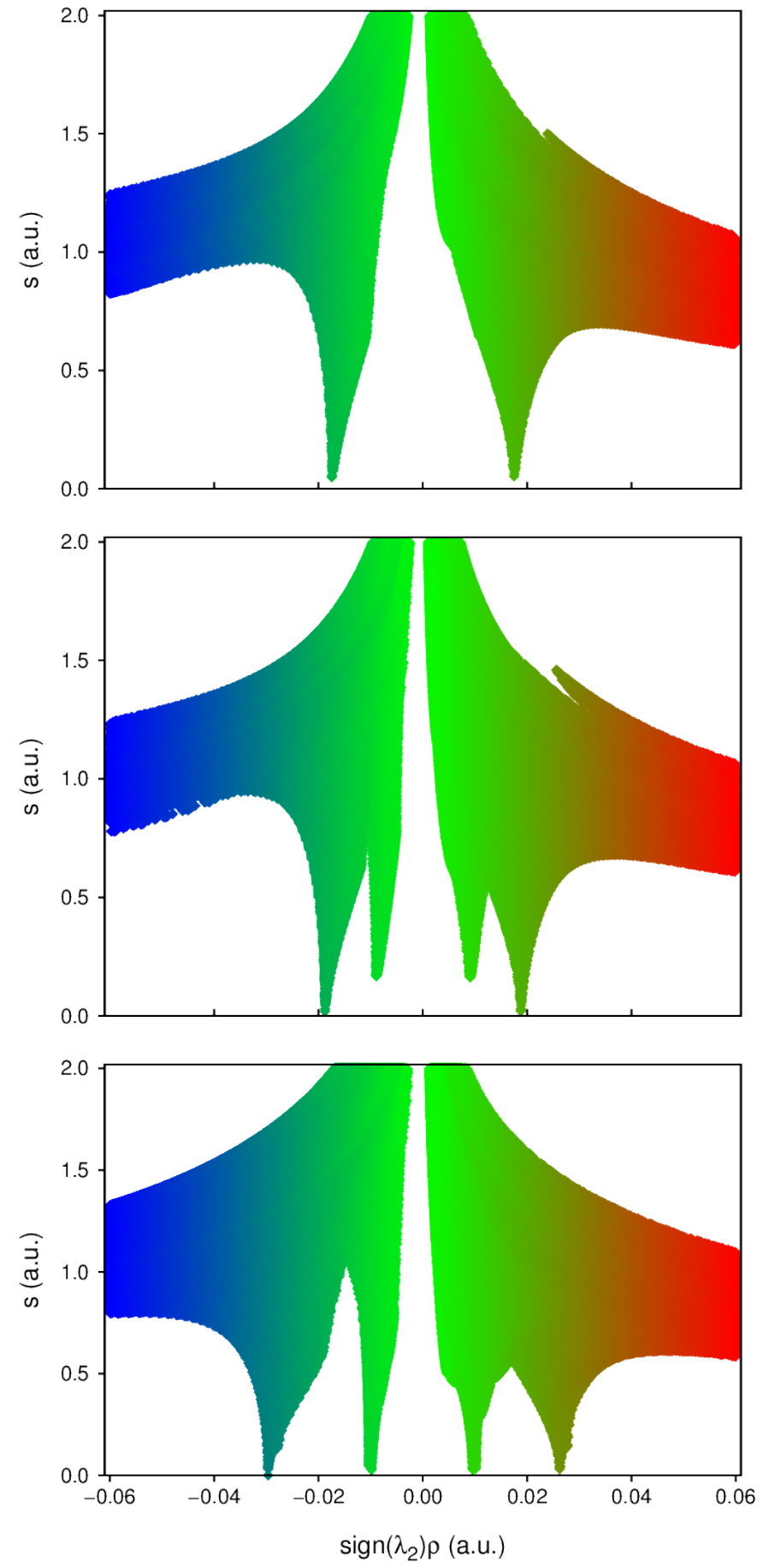

Figure S12: Plots of the reduced density gradient $s$ and $\operatorname{sign}\left(\lambda_{2}\right) \rho$ for AE (top), BMAE (middle) and BFMAE (bottom) obtained with the CCSD(T)-F12a/cc-pVDZ-F12 method using the M06-2x/aug-cc-pVTZ optimised geometry. 

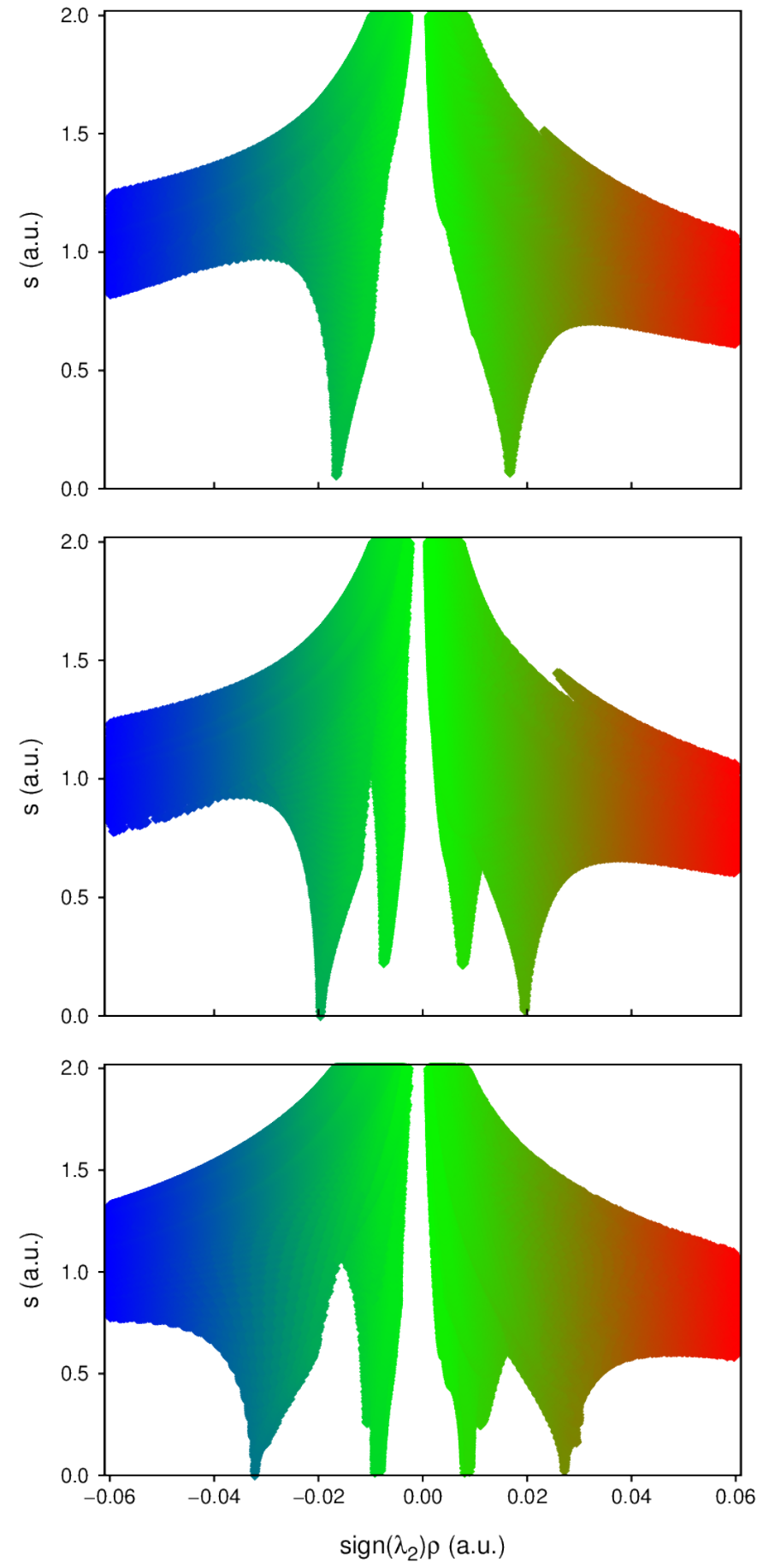

Figure S13: Plots of the reduced density gradient $s$ and $\operatorname{sign}\left(\lambda_{2}\right) \rho$ for AE (top), BMAE (middle) and BFMAE (bottom) obtained with the CCSD(T)-F12a/cc-pVDZ-F12 method using the B3LYP/aug-cc-pVTZ optimised geometry. 

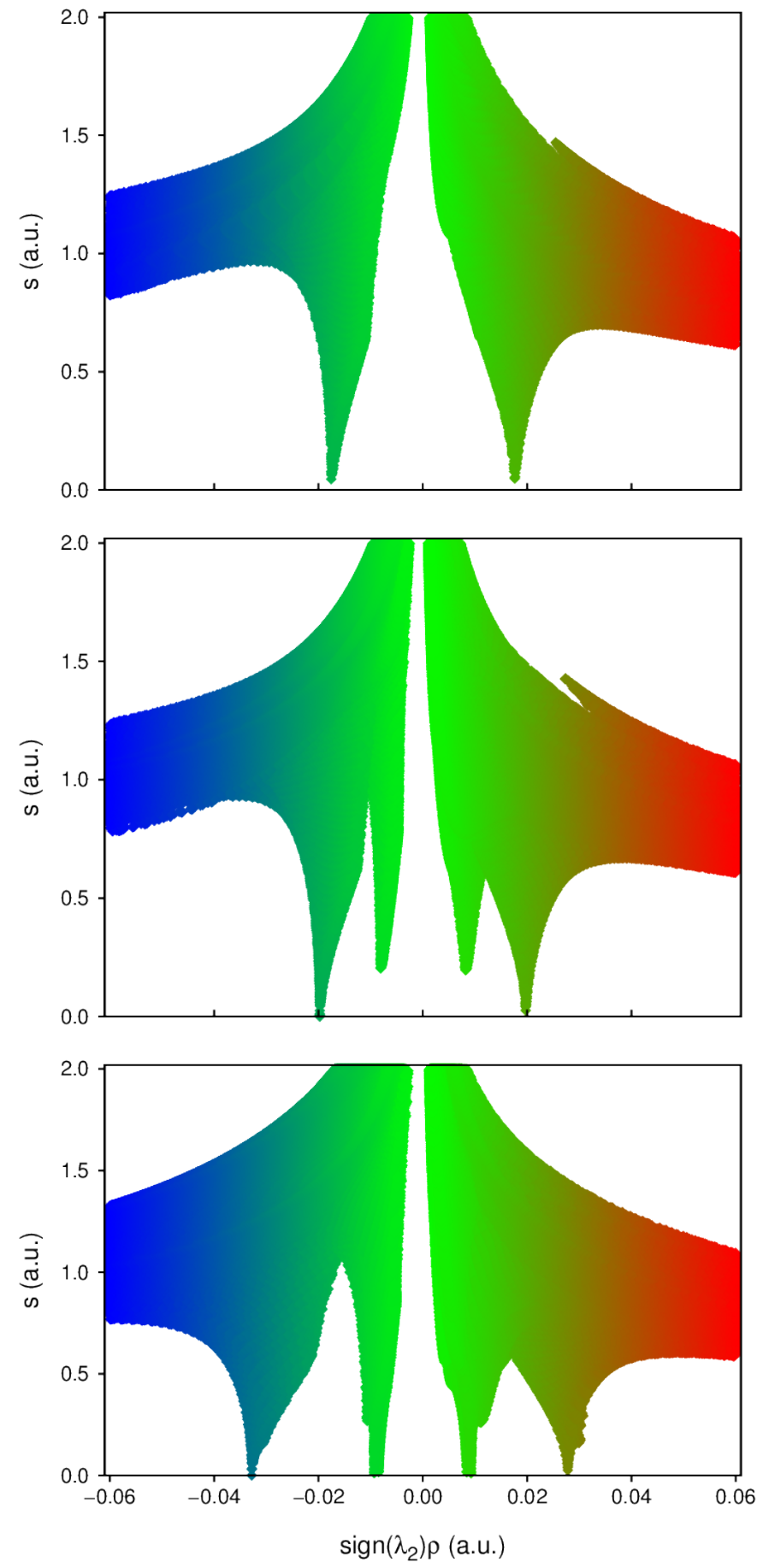

Figure S14: Plots of the reduced density gradient $s$ and $\operatorname{sign}\left(\lambda_{2}\right) \rho$ for AE (top), BMAE (middle) and BFMAE (bottom) obtained with the CCSD(T)-F12a/cc-pVDZ-F12 method using the WB97XD/aug-cc-pVTZ optimised geometry. 

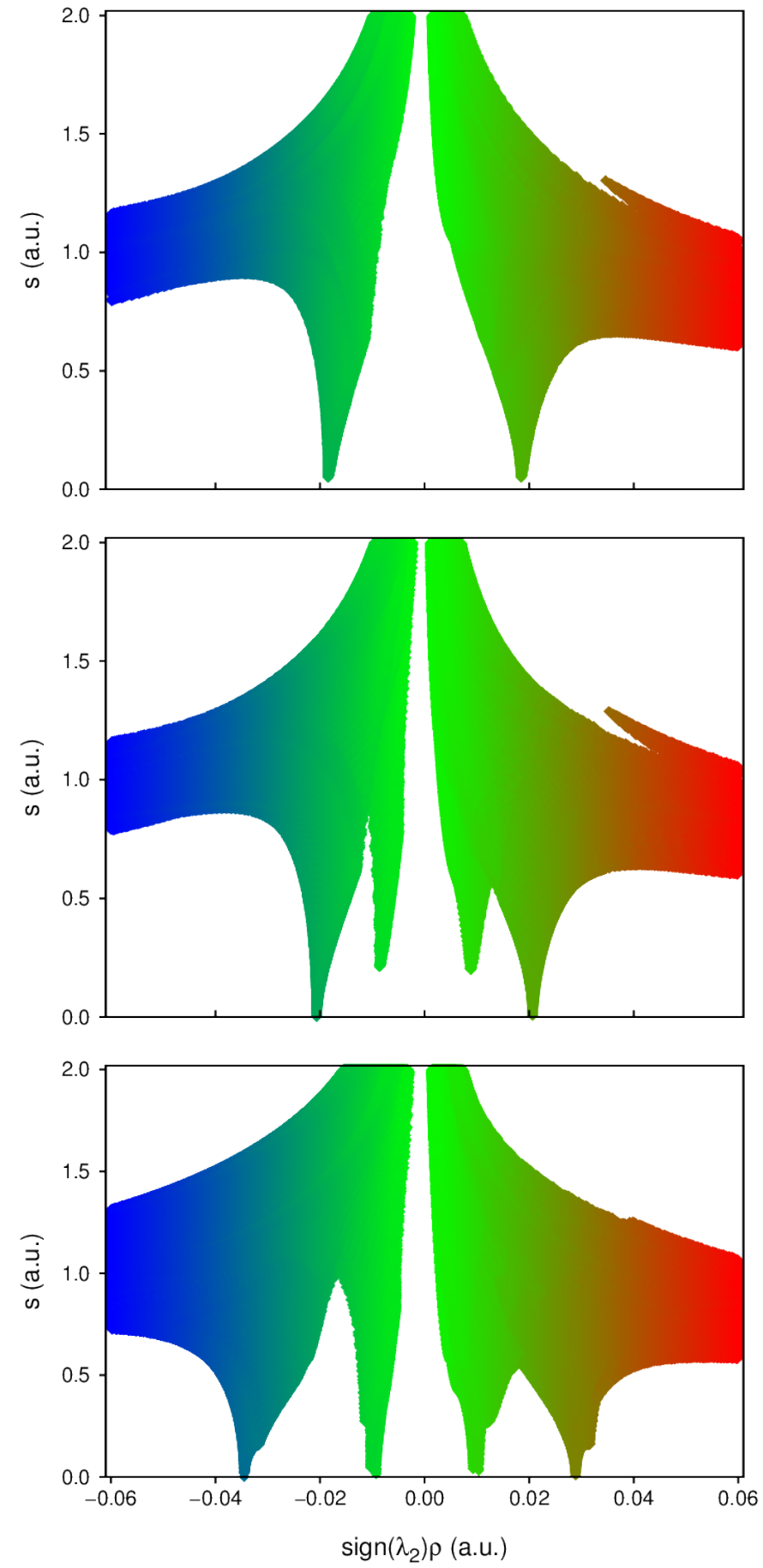

Figure S15: Plots of the reduced density gradient $s$ and $\operatorname{sign}\left(\lambda_{2}\right) \rho$ for AE (top), BMAE (middle) and BFMAE (bottom) obtained with the wB97XD/aug-cc-pVTZ method and optimised geometry. 

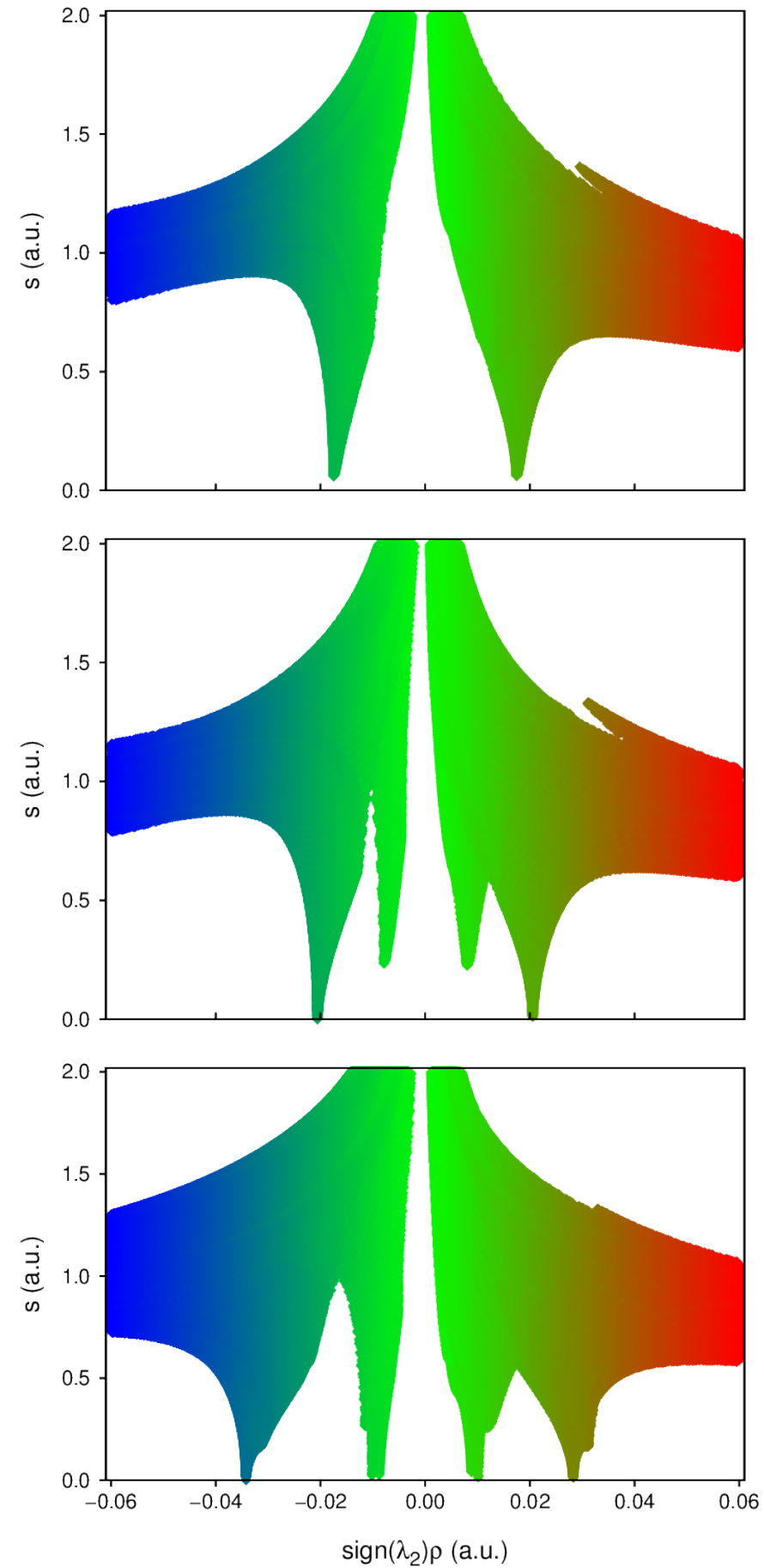

Figure S16: Plots of the reduced density gradient $s$ and $\operatorname{sign}\left(\lambda_{2}\right) \rho$ for AE (top), BMAE (middle) and BFMAE (bottom) obtained with the B3LYP/aug-cc-pVTZ method and optimised geometry. 

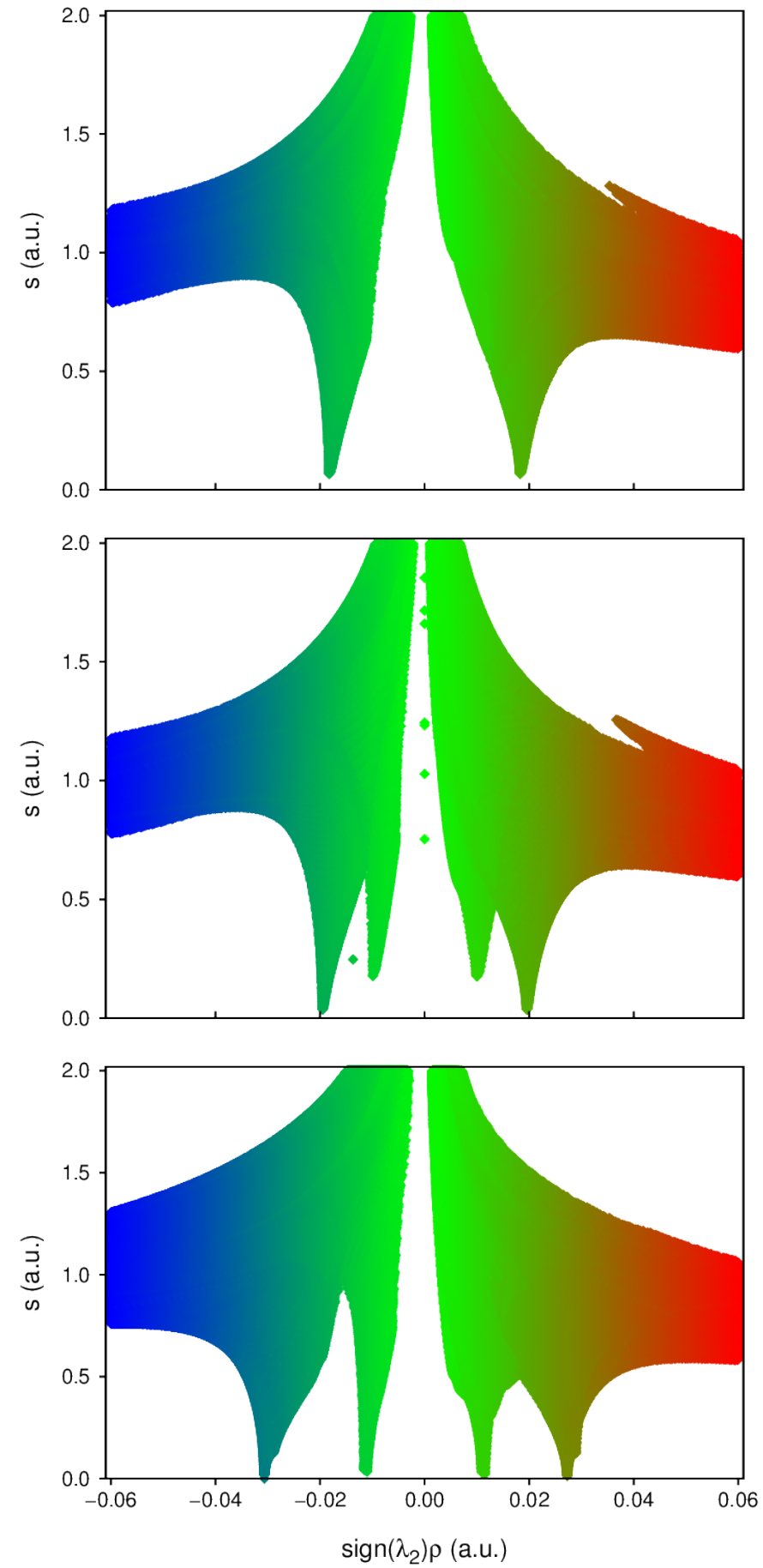

Figure S17: Plots of the reduced density gradient $s$ and $\operatorname{sign}\left(\lambda_{2}\right) \rho$ for AE (top), BMAE (middle) and BFMAE (bottom) obtained with the M06-2x/aug-cc-pVTZ method and optimised geometry. 

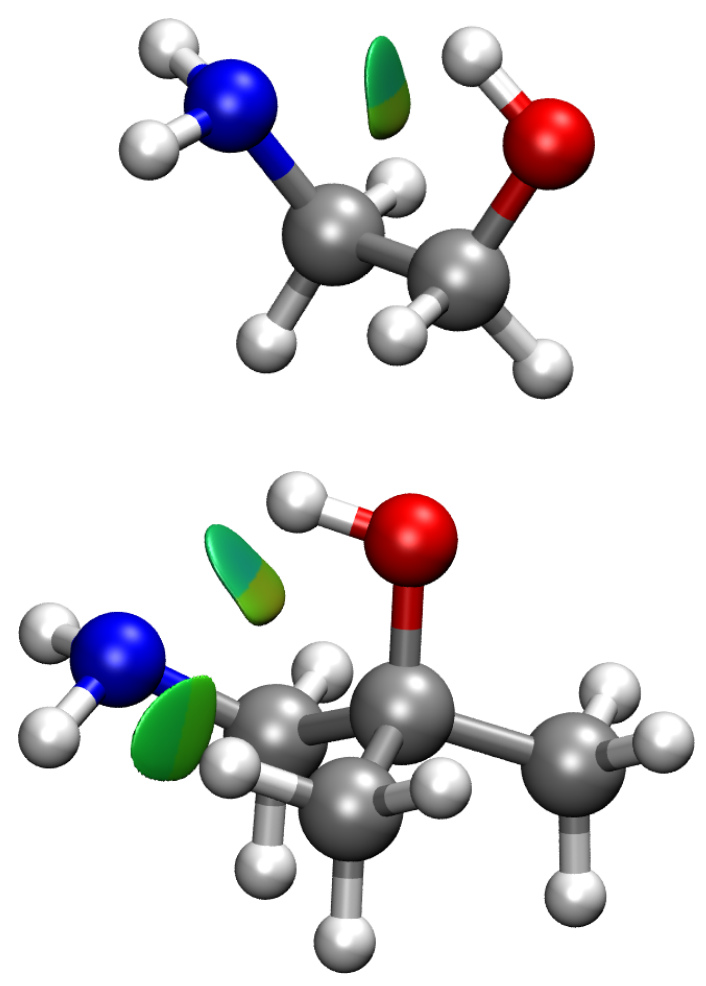

Figure S18: NCI isosurfaces for AE (top) and BMAE (bottom) obtained with the CCSD(T)F12a/cc-pVDZ-F12 method. $s=0.5$ a.u. and a blue-green-red colour scale from $-0.06<\rho<$ $+0.06$

\section{References}

(1) Frisch, M. J.; Trucks, G. W.; Schlegel, H. B.; Scuseria, G. E.; Robb, M. A.; Cheeseman, J. R.; Scalmani, G.; Barone, V.; Mennucci, B.; Petersson, G. A. et al. Gaussian 09. Gaussian Inc., Wallingford, CT, 2009.

(2) Werner, H. J.; Knowles, P. J.; Knizia, G.; Manby, F. R.; Schütz, M. Molpro: A GeneralPurpose Quantum Chemistry Program Package. Wiley Interdiscip. Rev. Comput. Mol. Sci. 2012, 2, 242-253. 
(3) Hill, J. G.; Peterson, K. A.; Knizia, G.; Werner, H.-J. Extrapolating MP2 and CCSD explicitly correlated correlation energies to the complete basis set limit with first and second row correlation consistent basis sets. J. Chem. Phys. 2009, 131, 194105.

(4) Zou, W. Molden2AIM. Southern Methodist University, Dallas, TX. 

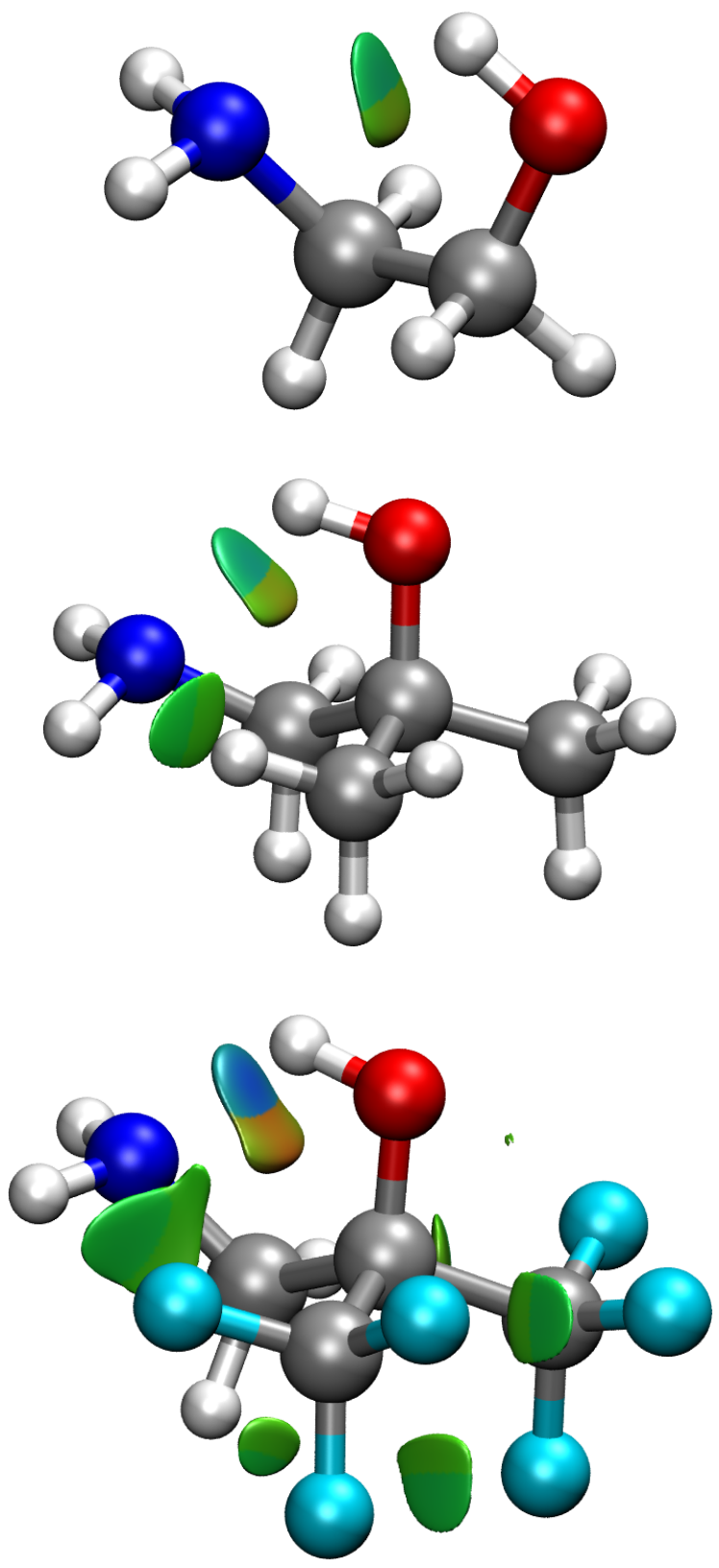

Figure S19: NCI isosurfaces for AE (top), BMAE (middle) and BFMAE (bottom) obtained with the B3LYP/aug-cc-pVTZ method and optimised geometry. $s=0.5$ a.u. and a bluegreen-red colour scale from $-0.06<\rho<+0.06$ 

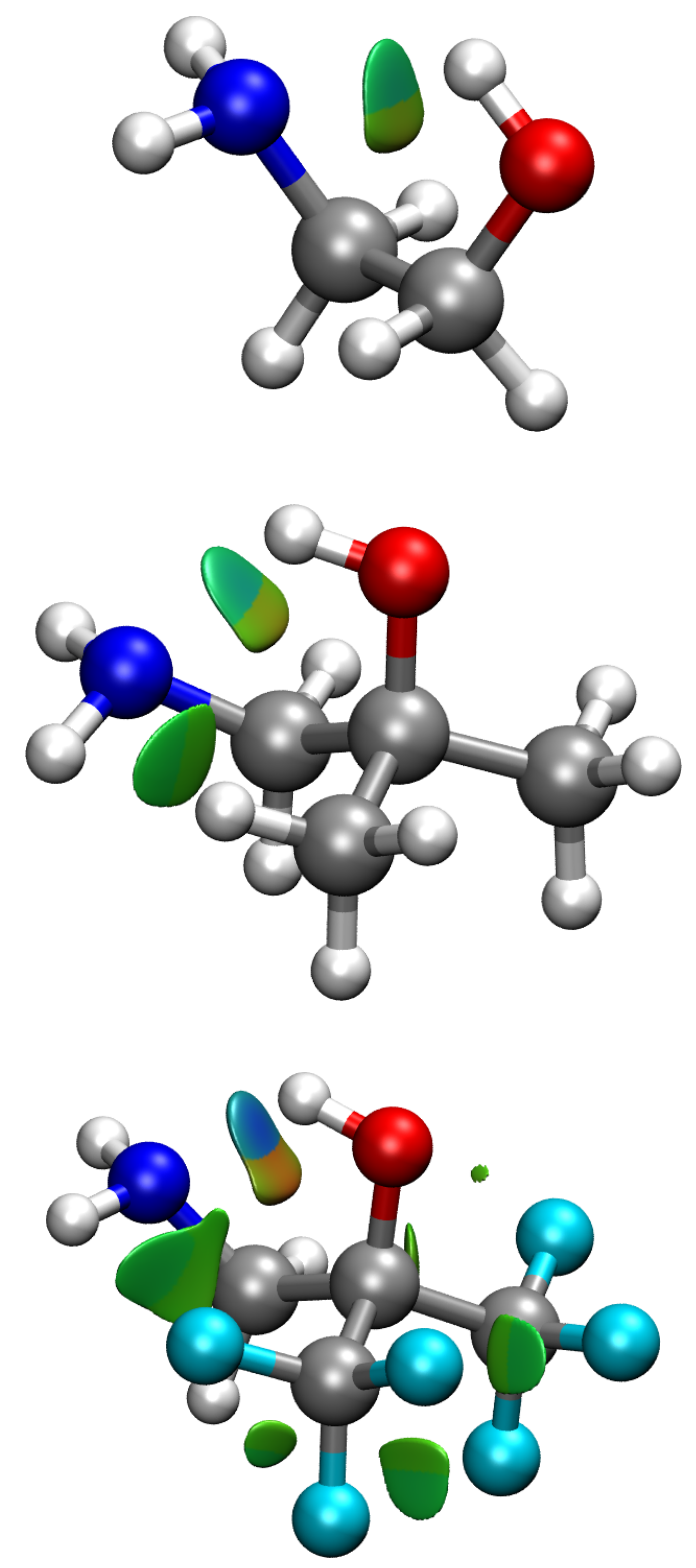

Figure S20: NCI isosurfaces for AE (top), BMAE (middle) and BFMAE (bottom) obtained with the WB97XD/aug-cc-pVTZ method and optimised geometry. $s=0.5$ a.u. and a bluegreen-red colour scale from $-0.06<\rho<+0.06$ 

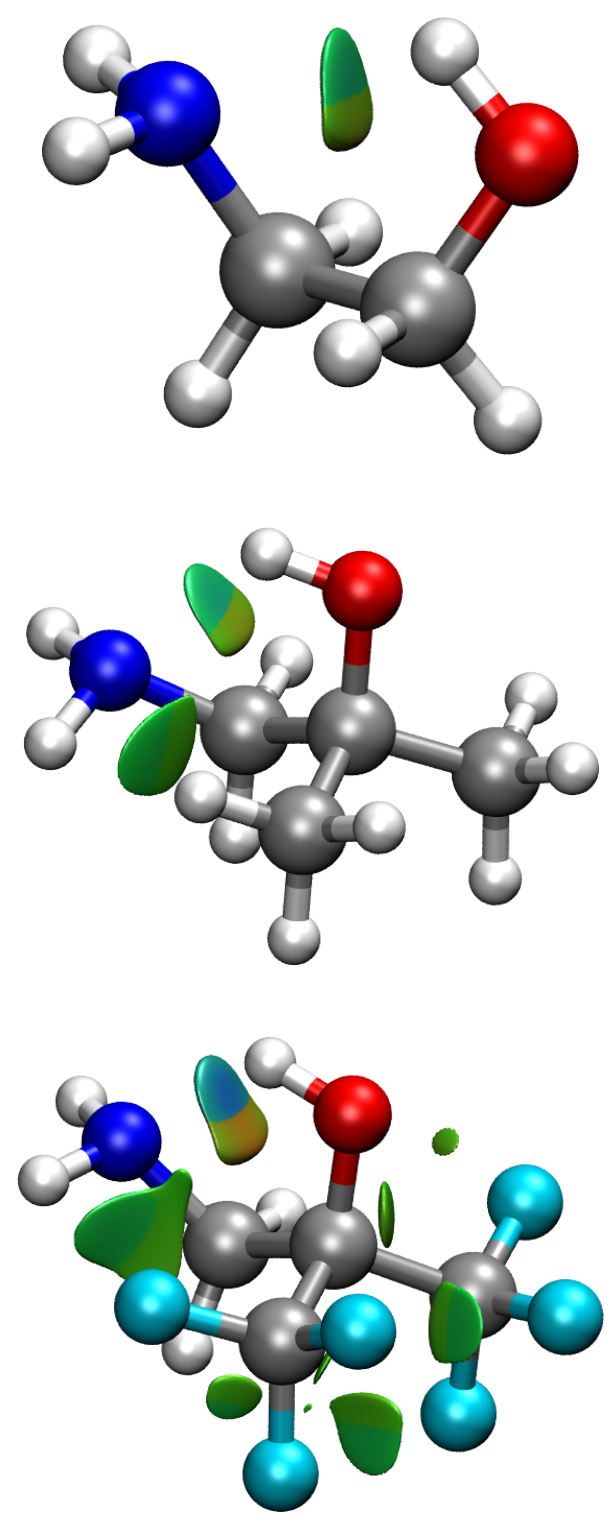

Figure S21: NCI isosurfaces for AE (top), BMAE (middle) and BFMAE (bottom) obtained with the B3LYP/aug-cc-pVTZ method and optimised geometry. $s=0.5$ a.u. and a bluegreen-red colour scale from $-0.06<\rho<+0.06$ 

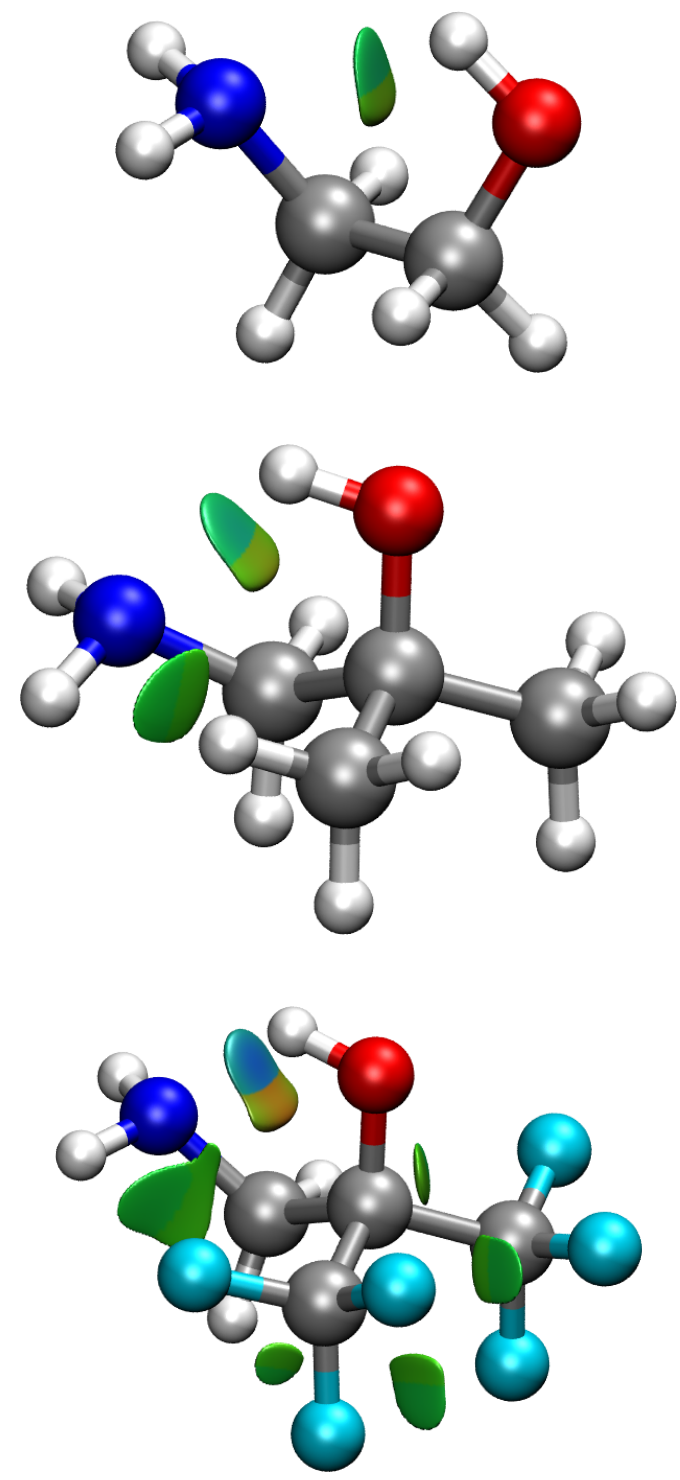

Figure S22: NCI isosurfaces for AE (top), BMAE (middle) and BFMAE (bottom) obtained with the CCSD(T)-F12a/cc-pVDZ-F12 method using the B3LYP/aug-cc-pVTZ optimised geometry. $s=0.5$ a.u. and a blue-green-red colour scale from $-0.06<\rho<+0.06$ 

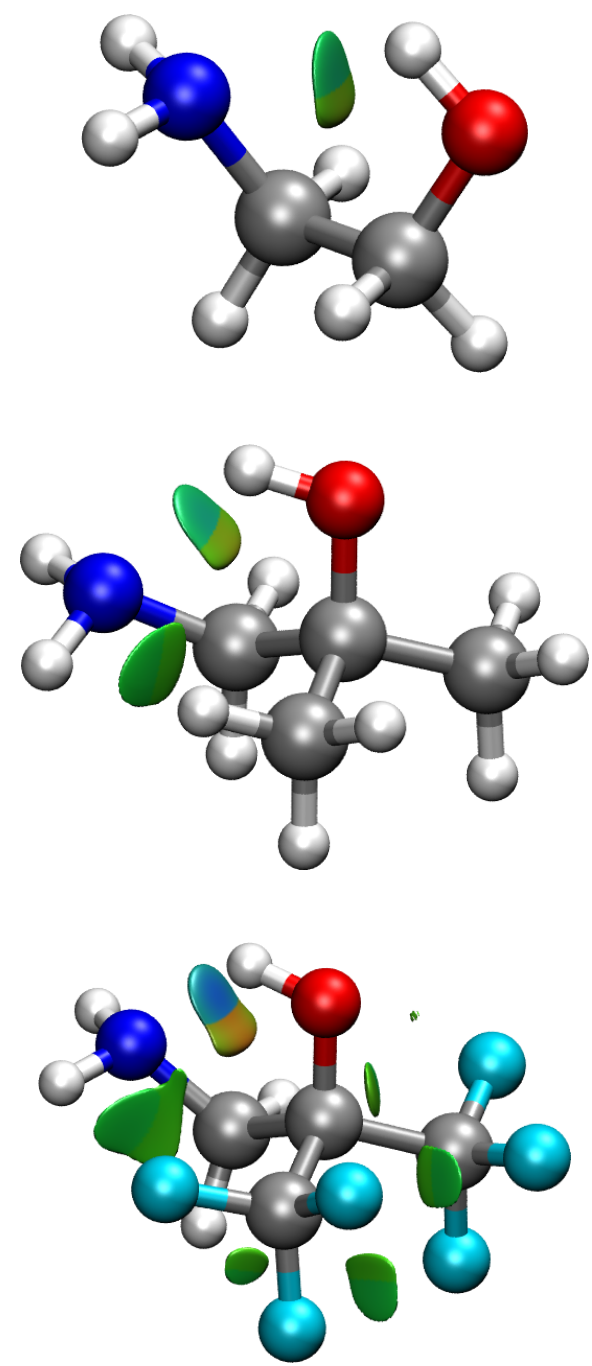

Figure S23: NCI isosurfaces for AE (top), BMAE (middle) and BFMAE (bottom) obtained with the CCSD(T)-F12a/cc-pVDZ-F12 method using the WB97XD/aug-cc-pVTZ optimised geometry. $s=0.5$ a.u. and a blue-green-red colour scale from $-0.06<\rho<+0.06$ 

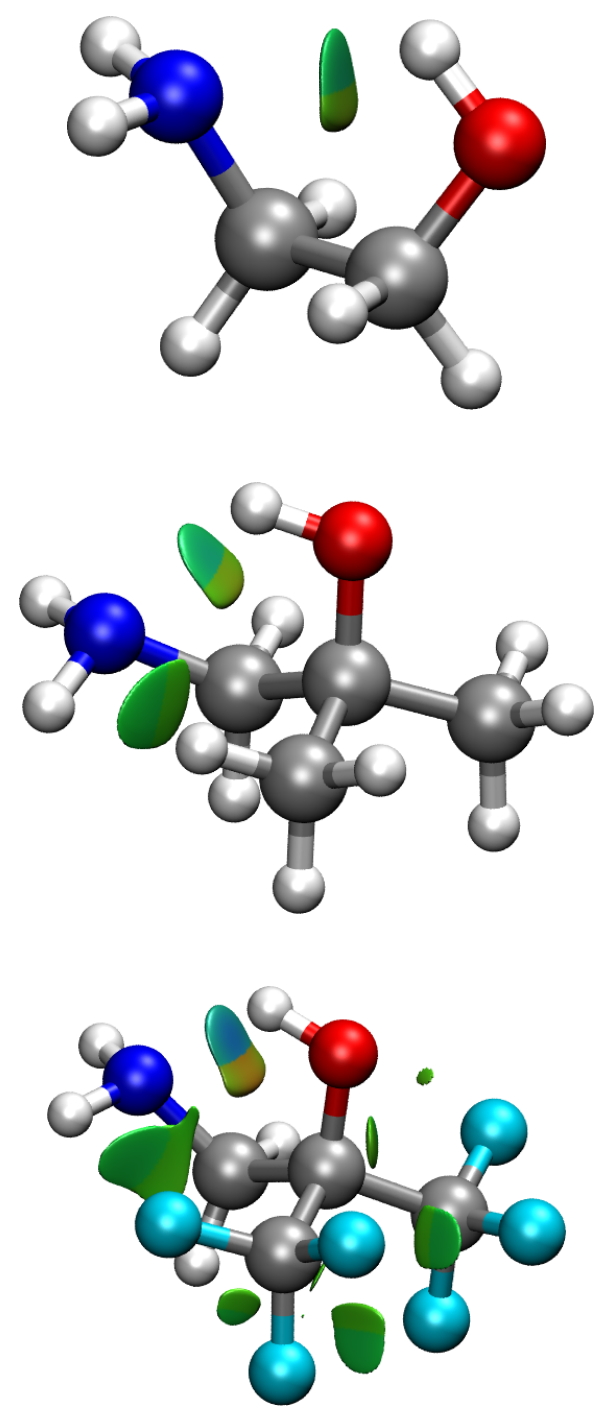

Figure S24: NCI isosurfaces for AE (top), BMAE (middle) and BFMAE (bottom) obtained with the CCSD(T)-F12a/cc-pVDZ-F12 method using the M06-2x/aug-cc-pVTZ optimised geometry. $s=0.5$ a.u. and a blue-green-red colour scale from $-0.06<\rho<+0.06$ 